\title{
CURRENT STATUS OF THE SSRF PROJECT
}

\author{
S. Y. Chen, H. J. Xu, and Z. T. Zhao ${ }^{\dagger}$, SSRC, Shanghai 201800, P. R. China
}

\begin{abstract}
The Shanghai Synchrotron Radiation Facility (SSRF) is an intermediate energy light source under design and R\&D at Shanghai National Synchrotron Radiation Center (SSRC). It is based on a $3.5 \mathrm{GeV}$ electron storage ring with a circumference of $396 \mathrm{~m}$, a beam current of 200 $300 \mathrm{~mA}$ and a beam emittance of $5-12 \mathrm{~nm} \cdot \mathrm{rad}$. Since its R\&D began in 1999, the design of the SSRF has been evolved smoothly towards a cost-effective machine required by its future users, and the prototypes of its key components have been manufactured and tested up to the design specifications. This paper reviews the design and $\mathrm{R} \& \mathrm{D}$ progress of the SSRF project and presents its current status.
\end{abstract}

\section{INTRODUCTION}

The SSRF was proposed by the Chinese Academy of Sciences and Shanghai Municipal Government in 1995, one year after that the SSRF Conceptual Design was completed and formally reviewed by an international review committee. The R\&D program for SSRF project was approved with a budget of $80 \mathrm{M}$ Chinese Yuan in 1998 and was conducted at SSRC from January 1999 to March 2001. During this period, 41 prototypes of the main components of the SSRF accelerators and beam lines have been developed and tested up to their design specifications. In the mean time, the SSRF technical design has been carried out and timely reviewed by the international review committees and the SSRF Committee of Science and Technology.

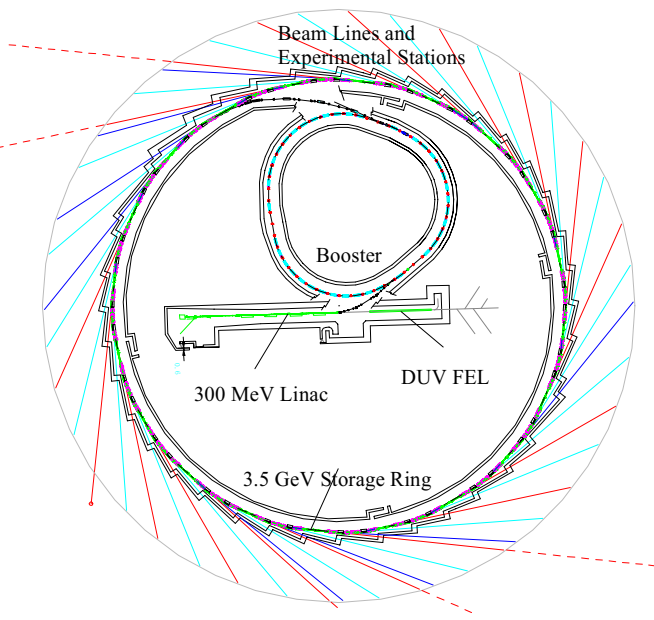

Fig.1 Layout of the SSRF

The SSRF complex sketched in Fig. 1 consists of three

†Email: zhaozt@ssrc.ac.cn main parts: a full energy injector including a $300 \mathrm{MeV}$ linac and a $3.5 \mathrm{GeV}$ booster, a $3.5 \mathrm{GeV}$ storage ring and the synchrotron radiation experimental facilities. The SSRF has been designed to produce high brightness and high flux X-ray in the photon energy range of $0.1 \sim 40 \mathrm{keV}$, with the emphasis on $5 \sim 20 \mathrm{keV}$. The current design of the SSRF storage ring does not pursue extreme low beam emittance but cost-effectiveness due to the user demands. The SSRF storage ring energy of $3.5 \mathrm{GeV}$ is determined by following the concept of using intermediate energy beam and advanced insertion devices to get the radiation for majority of the SR users.

Through a biding process among 5 candidates, the Zhang-Jiang High-Tech Park in Pudong, Shanghai has been chosen to site the SSRF complex. The SSRF site is a $600 \mathrm{~m} \times 300 \mathrm{~m}$ green land and readily accessible now.

\section{STORAGE RING}

The storage ring is the core part of the SR light source. Its characteristics determine the source performance. The design of the SSRF storage ring has been evolved to a cost effective machine over the last 5 years [1] [2], its current specifications are featured with robust and flexible lattice configuration and advanced mature technology. Apart from brightness and flux, other essential issues of the storage ring are related to beam stability, such as ground conditions, girder vibrations, chamber mechanical stability and temperature control in the storage ring tunnel. Further more the top-up injection is also incorporated into the design considerations.

\subsection{Magnetic Lattice and Performances}

The latest SSRF storage ring lattice is composed of 20 double-bend achromat (DBA) cells with 396 meters in circumference. It provides ten $7.24 \mathrm{~m}$ straight sections and ten $5.0 \mathrm{~m}$ straight sections for accommodating injection components, RF cavities and insertion devices. Each asymmetrical DBA cell contains 2 bending magnets, 10 quadrupoles and 7 sextupoles. This lattice has normal beta and hybrid beta configurations, which can adjust the beta functions in the middle of the straight section in a wide range. The high $\beta_{x}$ is $12 \mathrm{~m}$ and the low $\beta_{x}$ is about $1 \mathrm{~m}$, the tunes $\left(\mathrm{Q}_{\mathrm{x}} / \mathrm{Q}_{\mathrm{y}}\right)$ are $18.81 / 8.77$ and $22.19 / 8.23$ respectively. The non-zero dispersion in straight section is also employed in the low emittance lattice configurations, which gives a reduction factor of about 2.4 to the natural emittance.

The dynamic aperture of each SSRF ring lattice configuration has been examined with RACETRACK and MAD, both of the high beta and hybrid beta lattices have reasonably larger dynamic aperture and momentum acceptance with the effects of systematic and random 
multipole errors of magnets. The influence of insertion devices upon dynamic aperture is also verified with and without magnet errors, the magnet errors dominate the ring dynamic aperture.

The main parameters of the SSRF ring are shown in Table 1. And the corresponding photon brightness is depicted in Fig.2.

Table 1. Main Parameters of the SSRF Storage Ring

\begin{tabular}{|c|c|}
\hline Energy $(\mathrm{GeV})$ & 3.5 \\
\hline Circumference (m) & 396 \\
\hline Harmonic Number & 660 \\
\hline Nature Emittance (nm.rad) & $4.6 \sim 12.1$ \\
\hline Beam Current, Multi-Bunch (mA) & $200 \sim 300$ \\
\hline Single-Bunch (mA) & $>5$ \\
\hline Straight Lengths (m) & $10 \times 7.24,10 \times 5$ \\
\hline Betatron tunes, $\mathrm{Q}_{\mathrm{x}} / \mathrm{Q}_{\mathrm{y}}$ & $\begin{array}{r}18.81 / 8.77 \\
22.19 / 8.23\end{array}$ \\
\hline Momentum Compaction & $6.9 \times 10^{-4}$ \\
\hline RF Frequency (MHz) & 499.65 \\
\hline RF Voltage (MV) & 4 \\
\hline Dipole Radiation per Turn (MeV) & 1.256 \\
\hline Bunch Length $(\mathrm{mm})$ & 4.59 \\
\hline Beam Lifetime (hrs) & $>20$ \\
\hline
\end{tabular}

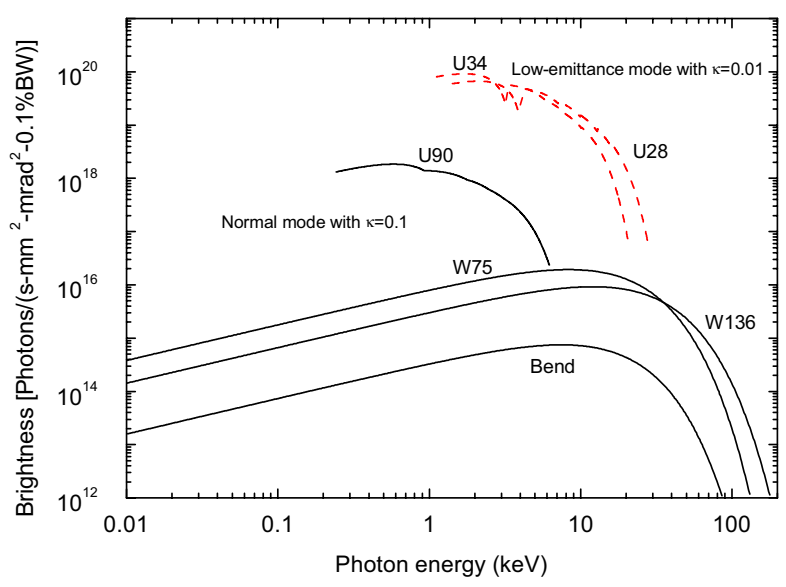

Fig.2 Spectral Brightness of SSRF

The new developments in insertion devices, such as high harmonic operation, in-vacuum IDs, mini-gap IDs and etc., will enhance the SSRF performance. The photon brightness of the SSRF sketched above can be further improved by employing advanced insertion devices.

\subsection{Magnet System}

The SSRF storage ring has $40 \mathrm{C}$-shape straight dipoles, 200 Collins-type quadrupoles, $140 \mathrm{C}$-shape sextupoles and 78 horizontal and vertical combined dipole correctors. All magnets have been designed at SSRC, the $1.66 \mathrm{~m}$ dipole has a good field region of $\pm 40.3 \mathrm{~mm}$ in horizontal plane for accommodating the $32.6 \mathrm{~mm}$ beam sagitta. The $56 \mathrm{~mm}$ dipole gap, $72 \mathrm{~mm}$ quadrupole diameter and $88 \mathrm{~mm}$ sextupole diameter were determined by the required minimum beam chamber aperture. The dipole field is $1.105 \mathrm{~T}$, the maximum quadrupole gradient is $18.5 \mathrm{~T} / \mathrm{m}$ and the maximum sextupole gradient is $500 \mathrm{~T} / \mathrm{m}^{2}$. The prototypes of a dipole, a quadrupole and a sextupole have been built and magnetically measured up to the specifications. Their corresponding harmonic contents of the integral fields in the good field regions, normalised to the main field components, are less than $4 \times 10^{-4}, 5 \times 10^{-4}$ and $3 \times 10^{-3}$ respectively.

One SSRF ring arc contains 3 six-strut type girders. Each girder supports one vacuum chamber with equivalent length. A $5.8 \mathrm{~m}$ long girder was designed and built in Shanghai with adjusting precision of $0.01 \mathrm{~mm}$ in three directions. This girder's lowest frequencies were measured with $5.88 \mathrm{~Hz}, 6.88 \mathrm{~Hz}$ and $27 \mathrm{~Hz}$ to longitudinal, horizontal and vertical vibration modes.

\subsection{Vacuum System}

The SSRF ring vacuum system is designed to reach the mean dynamic pressure less than 1ntorr at beam current of $300 \mathrm{~mA}$. Each storage ring arc has 3 machined antechamber sections made of aluminium A5083-H321, 3 horizontal and 5 vertical photon absorbers as well as 2 bellows. The prototypes of the $6 \mathrm{~m}$ long antechamber, the RF shielded bellows, the copper absorbers and the TSP and NEG pumps were designed and constructed during the last two years. A $6 \mathrm{~m}$ antechamber vacuum system was assembled at SSRC with vacuum pumped down to 0.05 ntorr by TSP. The PSD test of the horizontal photon absorber was carried out on a KEK PF beam line, and the bellow was tested with high RF power at KEK.

\subsection{Injection System}

The storage ring injection system has 4 kickers, 1 eddy current septum and 1 DC septum. Two kickers at both ends of the injection chain are located in the neigboring achromatic arcs across the dipoles. The DC septum, the eddy current septum and two central kickers are placed in a $7.24 \mathrm{~m}$ straight section. In the SSRF R\&D phase a kicker system including kicker magnet and its pulser was prototyped, the window frame type kicker magnet with a $\mathrm{Ni}-\mathrm{Zn}$ ferrite core in air produces $0.12 \mathrm{~T}$ peak field in amplitude and $4 \mu$ s bottom width in half-sine-wave pulse, its jitter is less than $\pm 6.5 \mathrm{~ns}$. The eddy-current septum and its $60 \mu$ s half-sine-wave pulser have been developed. Its main field peak is $0.62 \mathrm{~T}$ with the stability better than $\pm 0.1 \%$. The peak strength of stray field on the bump orbit is less than $0.02 \%$ of main field.

\subsection{Magnet Power Supplies}

There are more than 480 various power supplies for the SSRF storage ring magnets. All the main power supplies have been designed at SSRC, and the power supply prototypes of dipoles, quadrupoles and sextupoles were developed at SSRC. A dipole power supply prototype was tested with the stability of $\pm 1 \times 10^{-5} / 24 \mathrm{hrs}$ and the output current ripple less than $6 \times 10^{-6}$. The switch mode power 
supplies for quadrupoles and sextupoles were also tested up to their specifications.

\subsection{RF System}

In the current RF system design, 8 normal conducting $\mathrm{RF}$ cavities are used to provide $4 \mathrm{MV} \mathrm{RF}$ voltage to the beam, each cavity will be powered by a $180 \mathrm{~kW}$ klystron power amplifier. In the R\&D phase a cold test copper cavity was designed and fabricated with spin forming and electron beam welding techniques. And a high power RF system has been established, which contains a klystron and its power supply from THALES and THOMCAST, a circulator from AFT, a copper cavity borrowed from PF/KEK and low level RF control loops developed at SSRC. The high power RF system and the low level control loops have been tested separately. The high power test of the whole system with PF cavity and the control loops is under way. Recently the superconducting RF system for SSRF is being considered in cooperation with the BEPC-II project of IHEP in Beijing.

\section{INJECTOR}

The SSRF injector comprises a $300 \mathrm{MeV}$ electron linac followed by a $1 \mathrm{~Hz}$ booster, which accelerates the electrons to $3.5 \mathrm{GeV}$. As pre-injector, the linac has two operation modes: single bunch mode with pulse length of $1 \mathrm{~ns}$ and pulse current of $1.2 \mathrm{~A}$, or alternatively multibunch mode with pulse length of 300ns and macro pulse current of $180 \mathrm{~mA}$. In addition, the linac is also designed as an injector for the proposed DUV FEL facility, and the energy of this linac is therefore chosen for this purpose.

The full energy booster with circumference of $158.4 \mathrm{~m}$ has 24 FODO cells in 3 super periods. This kind of configuration makes the injection, RF acceleration and extraction separate from each other in space, therefore easier for mechanical installation. The main parameters of the SSRF booster are listed in Table 2.

Table 2. Main Parameters of the Booster

\begin{tabular}{|c|c|c|}
\hline \multicolumn{2}{|c|}{ Injection Energy ( $\mathrm{MeV})$} & 300 \\
\hline \multicolumn{2}{|c|}{ Output Energy (GeV) } & 3.5 \\
\hline \multicolumn{2}{|c|}{ Circumference $(\mathrm{m})$} & 158.40 \\
\hline \multicolumn{2}{|c|}{ Natural Emitance (nm·rad) } & $203(@ 3.5 \mathrm{GeV})$ \\
\hline \multirow{2}{*}{$\begin{array}{r}\text { Beam Current } \\
(\mathrm{mA})\end{array}$} & Single Bunch & 0.5 \\
\hline & Multi Bunch & 20 \\
\hline \multicolumn{2}{|c|}{ Repetition Rate $(\mathrm{Hz})$} & 1 \\
\hline \multicolumn{2}{|c|}{ RF Frequency (MHz) } & 499.65 \\
\hline \multicolumn{2}{|c|}{ RF Voltage (MV) } & 2.0 \\
\hline \multicolumn{2}{|c|}{ Energy Loss per Turn (MeV) } & 1.159 \\
\hline \multicolumn{2}{|c|}{ Super period Number } & 3 \\
\hline \multicolumn{2}{|c|}{ FODO Cell Number } & 24 \\
\hline \multicolumn{2}{|c|}{ Cell Length $(\mathrm{m})$} & 6.600 \\
\hline \multicolumn{2}{|c|}{ Betatron Tunes } & $7.253 / 4.214$ \\
\hline \multicolumn{2}{|c|}{ Synchrotron Tune } & 0.0219 \\
\hline \multicolumn{2}{|c|}{ Momentum Compaction } & 0.02443 \\
\hline \multicolumn{2}{|c|}{ Bunch Length $(\mathrm{cm})$} & 2.46 \\
\hline
\end{tabular}

During the last two years, a $100 \mathrm{kV}$ DC electron gun, a pre-buncher and a buncher were developed and tested up to their specifications. The prototypes of the booster dipoles, quadrapoles and sextupoles and their power supplies were constructed within their specification requirements. The $3 \mathrm{~m}$ stainless steel booster vacuum chamber was prototyped with satisfying performance both in vacuum and mechanical deformation.

\section{BEAMLINES}

Through close interaction with the existing and potential SR users in China, the following 7 beam lines for the first phase were selected from the 70 proposals by more than 20 universities and research institutes.

- Macromolecular Crystallography (ID)

- High Resolution X-ray Diffraction and Scattering

- X-ray Absorption Spectroscopy (ID)

- Hard X-ray Micro-Focusing

- Medical Application (ID)

- $\quad$ Soft X-ray Coherent Microscopy (ID)

- LIGA

In the SSRF R\&D phase, a front-end for the ID beamline and a double focusing monochromator were developed and tested off-line. A new type of long trace profiler has been built and used for the measurement of the crystal surface.

\section{CONCLUSION}

The SSRF specifications have been detailed with the completion of the technical design, and the prototypes made under the SSRF R\&D program are qualified for formal construction by minor modifications. While waiting for the final project approval of the central government, we are going to construct a $300 \mathrm{MeV}$ electron linac from the second half of this year.

\section{ACKNOWLEDGEMENTS}

The SSRF technical design and the R\&D program were supported by China National Development and Planning Committee, the Shanghai Municipal Government and the Chinese Academy of Sciences, and performed by the SSRF project team. We are very much indebted to the members of the SSRF project team for their dedication and hard work. We are also grateful for the contributions and support from many experts in Japan, Europe and US, who gave their consultation and suggestions to the SSRF design and R\&D program.

\section{REFERENCES}

[1] S.Y. Chen and H.J. Xu, "Current Status of the Proposed Shanghai Synchrotron Radiation Facility," APAC'98, Tsukuba, March 23 - 27, 1998.

[2] S.Y. Chen, H.J. Xu and Z.T. Zhao, "Shanghai Synchrotron Radiation Facility," PAC'99, New York, March 27 - April 2, 1999. 